\title{
Dynamic cerebral autoregulation: different signal processing methods without influence on results and reproducibility
}

\author{
Erik D. Gommer $\cdot$ Eri Shijaku $\cdot$ Werner H. Mess • \\ Jos P. H. Reulen
}

Received: 29 April 2010/Accepted: 21 October 2010/Published online: 4 November 2010

(C) The Author(s) 2010. This article is published with open access at Springerlink.com

\begin{abstract}
Cerebral autoregulation controls cerebral blood flow under changing cerebral perfusion pressure. Standards for measurement and analysis of dynamic cerebral autoregulation (dCA) are lacking. In this study, dCA reproducibility, quantified by intraclass correlation coefficient, is evaluated for different methodological approaches of transfer function analysis (TFA) and compared with multimodal pressure flow analysis (MMPF). dCA parameters were determined in 19 healthy volunteers during three 15-min lasting epochs of spontaneous breathing. Every spontaneous breathing epoch was followed by $5 \mathrm{~min}$ of paced breathing at 6 cycles/min. These six measurements were performed in both a morning and an afternoon session. Analysis compared raw data pre-processing by mean subtraction versus smoothness priors detrending. The estimation of spectral density was either performed by averaging of subsequent time windows or by smoothing the spectrum of the whole recording. No significant influence of pre-processing and spectral estimation on dCA parameters was found. Therefore, there seems to be no need to prescribe a specific signal-processing regime. Poor reproducibility of gain and phase was found for TFA as well as for MMPF. Based on reproducibility, no preference can be made for morning versus afternoon measurements, neither
\end{abstract}

Electronic supplementary material The online version of this article (doi:10.1007/s11517-010-0706-y) contains supplementary material, which is available to authorized users.

E. D. Gommer $(\bowtie)$ · E. Shijaku · W. H. Mess · J. P. H. Reulen Department of Clinical Neurophysiology, University Hospital Maastricht, Maastricht, The Netherlands

e-mail: e.gommer@mumc.nl

E. D. Gommer

Department of Biomedical Engineering, University Hospital Maastricht, Maastricht, The Netherlands for spontaneous versus paced breathing. Finally, reproducibility results are not in favour of TFA or MMPF.

Keywords Cerebral blood flow - Autoregulation . Cerebral circulation - Transfer function analysis . Transcranial Doppler

\section{Introduction}

Under normal conditions, despite variations in cerebral perfusion pressure (CPP), cerebral blood flow is kept relatively constant in correspondence with the metabolic needs of the brain. The control mechanism that compensates for CPP variations is called cerebral autoregulation [1, 17, 22]. Various methods have been described to characterise this control system. A sudden step-wise decrease in arterial blood pressure (ABP) evokes a response, characterised by a few seconds lasting decreased flow followed by a relatively slow return to the base level. This cerebral blood flow velocity (CBFV)-response can be interpreted as a kind of step response of the control system and can be quantified by the so-called autoregulatory index (ARI) [28] ranging between 0 and 9. Another approach to quantify the dynamics of cerebral autoregulation $(\mathrm{dCA})[4,20,31]$ is transfer function analysis (TFA) of the presumed linear control system with $\mathrm{ABP}$ as the input and CBFV as the output signal. The transfer function gain and phase are estimated from the auto- and cross spectra of ABP and $\mathrm{CBFV}$ and the strength of the linear relation between $\mathrm{ABP}$ and CBFV is expressed in the system's coherence. From the step response function resulting from this transfer function, the system's ARI can be determined using least squares estimation. For each of the 10 ARI responses, the sum of squared differences with the TFA response is determined. 
A parabolic interpolation through these 10 values can be performed to estimate the value of ARI to one decimal space [21] at the minimum of the parabola.

A recently published and different approach to overcome some difficulties with Fourier-based spectral analysis is the multimodal pressure flow analysis (MMPF) [13, 18]. The authors showed that MMPF analysis may be less sensitive to problems with data non-stationarities and trends [13]. In a study on traumatic brain injury patients, better reproducibility was shown with MMPF phase compared to ARI [10]. Also non-linear approaches have been tried to evaluate dCA $[11,15]$, but clear benefits have not been shown yet.

A limiting factor in (clinical) use of quantitative dCA is the high variability observed in calculated parameters, such as gain, phase and ARI [5, 14, 21, 25]. This high variability is likely to limit reproducibility of these measurements. Brodie et al. showed poor ARI reproducibility with an intraclass correlation (ICC) below 0.5. It is unknown if reproducibility may be influenced by procedural and methodological options.

Currently, there is neither a procedural nor a methodological standard to compute dCA. A procedural aspect is the choice between using spontaneous [20,31] and induced blood pressure variations, e.g. by paced breathing at a frequency of 6 cycles/min $[9,24]$. Paced breathing requires cooperation of the patient, whereas spontaneous blood pressure variations can be recorded without specific instructions. The same holds for squat-stand manoeuvres, although this seems a promising addition [6]. Another procedural aspect concerns possible circadian variations in dCA. Ainslie et al. reported a significant lowering in ARI from evening to morning [3]. Therefore, it might be necessary to standardise the time of day for dCA recording.

For TFA, different signal processing techniques can be applied and the most common will be evaluated in this study. First, before applying Fourier transform, offset and/or slow trends are removed either by subtracting the mean or by sophisticated detrending procedures to remove also very low frequencies. Second, for spectral estimation, two approaches can be used: either the spectral averaging technique, also known as the Welch technique [30], in which spectra of subsequent epochs are averaged, or spectral smoothing in which the spectra are smoothed with triangular smoothing windows [29].

In this study, the effect on reproducibility will be evaluated for the different TFA analysis options and for the MMPF method. A major question is whether results of MMPF compared to TFA analysis are more consistent and show less variability. To reveal effects of the measurement conditions, also spontaneous versus paced breathing and morning versus afternoon recordings will be compared.

\section{Methods}

\subsection{Subjects and measurements}

Nineteen healthy subjects $(14 \mathrm{M} / 5 \mathrm{~F})$ between 18 and 53 years old (mean 28) voluntarily participated in the study. All were free of known cardiovascular, pulmonary and cerebrovascular disorders. Each subject was measured in supine position on two different days, one day in the morning at 10 a.m. and a different day one week apart in the afternoon at 2 p.m. The order of day was randomly assigned. Each recording session lasted about $1 \mathrm{~h}$. A session consisted of three successive runs each consisting of $15 \mathrm{~min}$ of spontaneous breathing followed by $5 \mathrm{~min}$ of 6 cycles/min frequency paced breathing.

A one channel electrocardiogram (ECG) was measured by an in-house made portable ECG-amplifier (IDEE Maastricht ${ }^{\circledR}$, The Netherlands). Continuous ABP was measured using a non-invasive finger blood pressure monitor (Portapres ${ }^{\circledR}$, TNO, Amsterdam, The Netherlands) commonly used in studies on dCA. The appropriate finger cuff (size: small, medium or large) was placed on the left-hand middle finger. During the recordings, the Physiocal procedure, an intermittently occurring calibration routine, was switched off. To measure CBFV simultaneously in the right- and left-middle cerebral artery, a Transcranial Dopplersonography device (DWL Multi-Dop ${ }^{\circledR}$ $\mathrm{X} 4$, Sipplingen, Germany) was used. The two 2-MHz probes were held in position by a special head frame.

\subsection{Data analysis}

ECG, ABP and right- and left-CBFV signals were recorded on a DAT-tape (TEAC-recorder) and thereafter offline digitised and stored on a $\mathrm{PC}$ with an in-house made acquisition and analysis software package (AFOnew version 2.33, IDEE Maastricht ${ }^{\circledR}$, The Netherlands). The sampling frequency for each signal was set to $250 \mathrm{~Hz}$. An automatic algorithm detected the ECG R-waves and determined the subsequent diastolic, mean and systolic blood pressure and corresponding end-diastolic, mean and peak-systolic CBFV. Artefacts were removed manually by linear interpolation. The beat-to-beat values of $\mathrm{ABP}$ and CBFV were resampled with $5 \mathrm{~Hz}$ using spline-interpolation [20]. From the ABP, the overall mean value was subtracted and the CBFVs were normalised with respect to the mean. This resulted in zero-mean signals suited for spectral analysis to estimate the transfer function. The transfer function was calculated by

$H(f)=\frac{S_{x y}(f)}{S_{x x}(f)}$,

where $S_{x x}(f)$ is the autospectrum of changes in $\mathrm{ABP}$ and $S_{x y}(f)$ is the cross-spectrum between the ABP- and CBFV- 
signals. The transfer function magnitude $|H(f)|$ and phase spectrum $\Phi(f)$ were derived from the real part $H_{\mathrm{R}}(f)$ and imaginary part $H_{\mathrm{I}}(f)$ of the complex transfer function as

$|H(f)|=\sqrt{\left[H_{\mathrm{R}}(f)\right]^{2}+\left[H_{\mathrm{I}}(f)\right]^{2}}$,

$\Phi(f)=\arctan \frac{H_{\mathrm{I}}(f)}{H_{\mathrm{R}}(f)}$.

The squared coherence function $\gamma^{2}(f)$ was estimated by

$\gamma^{2}(f)=\frac{\left|S_{x y}(f)\right|^{2}}{S_{x x}(f) S_{y y}(f)}$,

where $S_{y y}(f)$ is the autospectrum of changes in CBFV. The squared coherence reflects the strength of the linear relationship between $\mathrm{ABP}$ and $\mathrm{CBFV}$ for each frequency on a scale from 0 to 1 .

To reduce variance of the spectral estimates of the transfer function either spectral averaging or spectral smoothing can be performed. Spectral averaging was first introduced by Welch [30], and averaged spectra were calculated over multiple half overlapping epochs. An epoch length of $50 \mathrm{~s}$ is chosen to result in an adequate frequency resolution of at most $0.02 \mathrm{~Hz}$. Spectral smoothing reduces variance by filtering the spectrum with a triangular window averaging over a frequency band. In this case, the spectrum is calculated over almost the entire measurement resulting in a much finer frequency resolution. In this study, four different combinations of parameter settings (Table 1) for detrending were tested. In methods I and III, only mean subtraction was performed, whereas in methods II and IV smoothness priors detrending [27] with a $0.04-\mathrm{Hz}$ cut-off frequency $(\lambda=500)$ was used to remove very low frequency trends. Each data epoch was windowed with a Hanning-window. In methods I and II, half overlapping 51.2 -s long epochs of 256 data points with a $5-\mathrm{Hz}$ resampling rate were used; in methods III and IV, 2048 points, corresponding to a 409.6-s epoch length, were used. In methods I and II, the spectrum was smoothed with a 3point triangular moving average window corresponding to a full width half maximum bandwidth of $0.04 \mathrm{~Hz}$; in III and IV, 31 points wide triangular window, also corresponding to a bandwidth of $0.04 \mathrm{~Hz}$ [16, 29].

From the resulting transfer functions, mean coherence, phase and gain were calculated in the $0.04-0.16 \mathrm{~Hz}$ frequency range. The $0.04-\mathrm{Hz}$ lower border has been chosen because the limited duration of the recordings makes interpretation of results below this frequency difficult. The upper frequency is chosen to include the major frequency range of dCA. The choice of the $0.04-0.16 \mathrm{~Hz}$ frequency interval does not introduce new problems of standardization since in this paper all methodologies and protocols are mutually compared using this fixed interval. Furthermore, the graphical presentation of our results as a function of frequency allows estimation for other frequency bands.

Paced breathing analysis was only possible for combinations I and II (Table 1) because of the limited $5 \mathrm{~min}$ duration of these recordings.

Autoregulatory index was calculated from the first $7 \mathrm{~s}$ of the step response function using a previously described method [14]. The step response to calculate the ARI is obtained by integrating the impulse response, being the inverse Fourier transform of the estimated transfer function. From the ABP and CBFV waveforms, the critical closing pressure and RAP were determined per beat using linear regression [19]. Before regression, individual ABP and CBFV beat waveforms were aligned for maximum correlation. RAP $(\mathrm{mmHgs} / \mathrm{cm})$ is the reciprocal slope of the regression line.

\subsection{Coherence criterion}

To allow reliable interpretation of dCA results, coherence levels need to exceed a minimum level. This minimum level, above which coherence differs significantly from zero, depends on the degrees of freedom of the spectral estimation procedure (computation in Appendix-Supplementary material). Computation based on the degrees of freedom resulted in a minimum needed squared coherence level of 0.06 for the 15 -min spontaneous breathing episodes and of 0.2 for the 5 -min of 6 cycles/min breathing periods.

\subsection{Multimodal pressure flow analysis}

For MMPF, a recently improved method was used including ensemble empirical mode decomposition (EEMD) [12, 18]. To extract spontaneous oscillations in $\mathrm{ABP}$ and $\mathrm{CBFV}$, empirical mode decomposition was used

Table 1 Summary of parameter choices in transfer function estimation

\begin{tabular}{lllccc}
\hline Combination & Detrending & $\begin{array}{l}\text { Window length } \\
\text { (samples) }\end{array}$ & $\begin{array}{l}\text { Window } \\
\text { duration (s) }\end{array}$ & $\begin{array}{l}\text { Triangular smoothing } \\
\text { window width }\end{array}$ & $\begin{array}{l}\text { Smoothing } \\
\text { bandwidth (Hz) }\end{array}$ \\
\hline I & Mean subtraction & 256 & 51.2 & 3 & 0.04 \\
II & Smoothness priors & 256 & 51.2 & 3 & 0.04 \\
III & Mean subtraction & 2048 & 409.6 & 31 & 0.04 \\
IV & Smoothness priors & 2048 & 409.6 & 31 & 0.04 \\
\hline
\end{tabular}


to decompose $\mathrm{ABP}$ and $\mathrm{CBFV}$ signals into intrinsic modes. For the EEMD, this decomposition was repeated 100 times with added white noise having an amplitude of $10 \%$ of the standard deviation of the signal. The resulting 100 realizations of intrinsic mode functions were averaged per mode. Only components 3 and 4 were used for the analysis, since these were the only components with frequencies in the range from 0.04 to $0.16 \mathrm{~Hz}$. To compare the values for phase and gain with the results from the TFA, averages were taken over the whole measurement course. A recently modified transfer function analysis (MTFA) also was applied [13]. This method evaluates gain and phase with TFA when instead of the beat-to-beat ABP and CBFV signals, the intrinsic mode functions of the MMPF were used.

\subsection{Statistical analysis}

The non-parametric Wilcoxon-signed rank test was used to test for left/right and morning/afternoon differences for each method. Reproducibility was quantified by the intraclass correlation coefficient (ICC) using the generalisability theory [7, 26]. ICC, defined as the sum of variances except for repeated measurements components divided by the total variance, was calculated [23] using the GENOVA (version 2.2, 1984) program. Although ICC is specific to the population studied, it does give clear indications of inter-method reproducibility being a major purpose of this study.

\section{Results}

\subsection{Spontaneous blood pressure variations}

From all recorded data $(19$ subjects $\times 2$ sessions $\times 1 \mathrm{~h}=39 \mathrm{~h}$ ), only a single period of $20 \mathrm{~min}$ had to be excluded because of bad signal quality. Pairwise comparison of coherence, gain and phase parameters between left and right $\mathrm{CBFV}$ recordings did not reveal significant differences. Therefore, per individual and recording session, left and right side data were pooled for further analysis. Morning and afternoon dCA parameters for the four analysis methods are given in the Supplementary material (Table S1). MMPF results are presented for intrinsic mode functions 3 and 4. The mean frequencies of ABP and CBFV for these components 3 and 4 and their standard deviations are indicative for narrow-banded signals. Also the frequencies of intrinsic mode functions 3 and 4 match very well for $\mathrm{ABP}$ and $\mathrm{CBFV}$.

The listed values for std ABP and std CBFV represent the standard deviation over time of, respectively, the mean ABP and mean CBFV. For none of the methods, significant differences were found between morning and afternoon values.

In Figs. 1, 2 and 3, the group averages of coherence, gain and phase spectra are presented for spontaneous breathing episodes for four different analysis schemes. There is good correspondence between the curves of the four analysis methods, and also the MMPF values for phase are close to these curves at the corresponding frequencies for components 3 and 4. The MTFA values are also in good correspondence when taking into account that these are averages the $0.04-0.16 \mathrm{~Hz}$ frequency range.

\subsection{Paced 6 cycles/min breathing}

Paced breathing lasted $5 \mathrm{~min}$. For this reason, the 2048point spectral smoothing method was not applicable. In Table S2 of the Supplementary material, dCA parameters are presented for morning and afternoon for the two analysis methods. No significant differences were found between morning and afternoon. The group averages of the phase spectra are plotted in Fig. 4 for the paced breathing episodes for the two detrending methods. For the $0.1-\mathrm{Hz}$ frequency component, there is good correspondence between the TFA methods and the MMPF and MTFA method.

\subsection{Comparison of signal processing methods}

In total, 48 independent sample comparisons were made for the four different signal-processing methods in spontaneous breathing. Only for coherence, statistically significant differences could be shown. Coherence was significantly

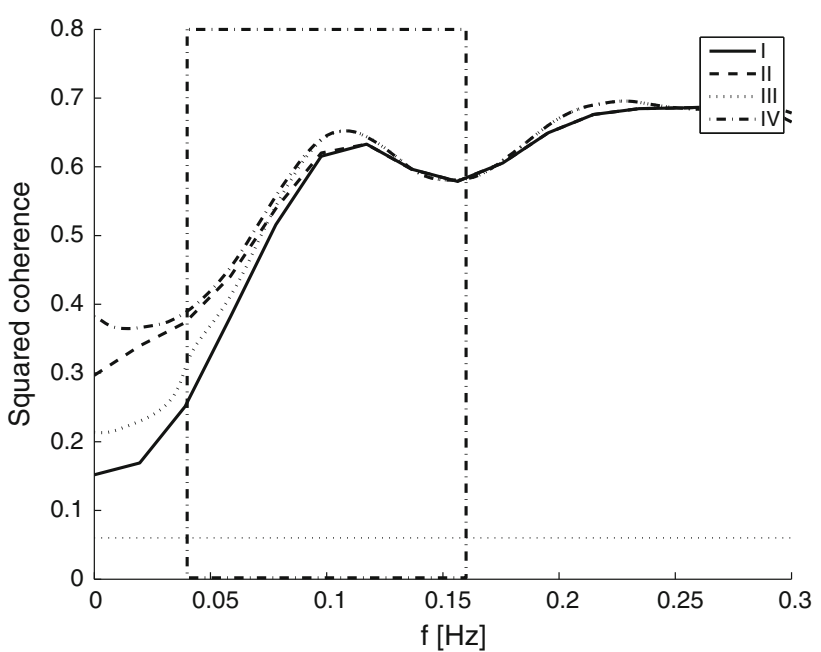

Fig. 1 Group-averaged spectral squared coherence plot for method I (solid), II (dashed), III (dotted) and IV (dash-dotted) during spontaneous breathing. Marked by the dashed box is the frequency range analysed. Marked by the dotted line is the coherence threshold value 


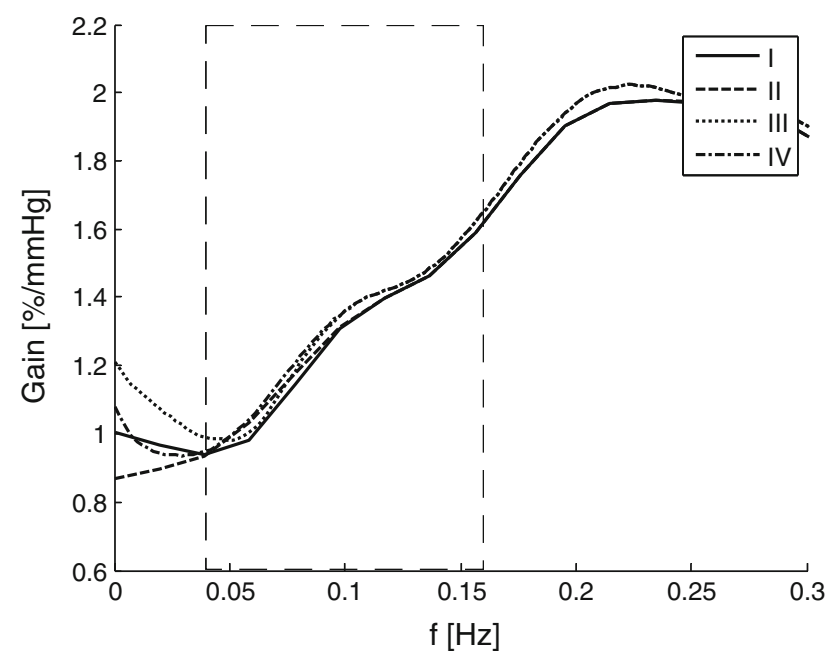

Fig. 2 Group-averaged spectral gain plot for method I (solid), II (dashed), III (dotted) and IV (dash-dotted) during spontaneous breathing. Marked by the dashed box is the frequency range analysed

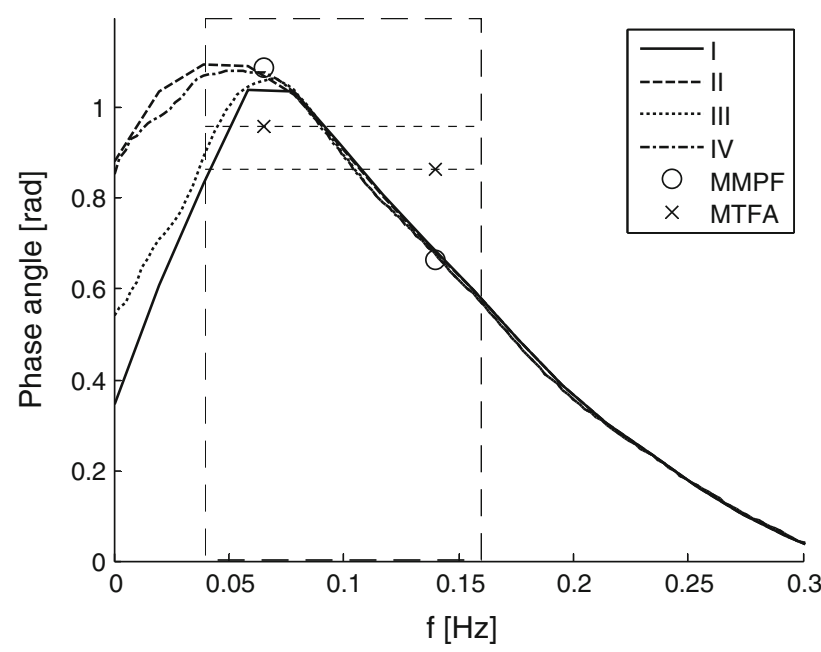

Fig. 3 Group-averaged spectral phase plot for TFA method I (solid), II (dashed), III (dotted) and IV (dash-dotted), MMPF method and MTFA method during spontaneous breathing. Marked by the dashed box is the frequency range analysed

lower for method I compared to III $(P=0.04)$ and IV $(P=0.005)$.

\subsection{Spontaneous versus paced breathing}

In total, 8 pairwise comparisons of dCA parameters for spontaneous versus paced breathing were performed. They showed that phase and ARI were significantly higher in paced versus spontaneous breathing using method II both for the morning $(P=0.001)$ and afternoon session $(P=0.01)$ and only in the morning $(P=0.006)$ for method I.

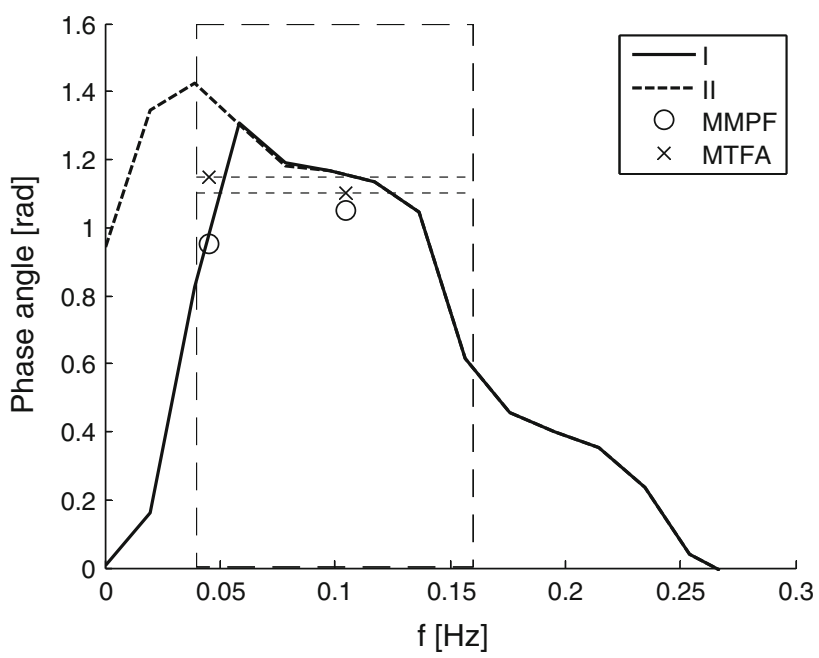

Fig. 4 Group-averaged spectral phase plot for TFA with (solid) and without (dashed) using smoothness priors detrending and MMPF and MTFA method during 6 cycles/min paced breathing. Marked by the dashed box is the frequency range analysed

\subsection{Reproducibility analysis}

As can be seen in the Tables S3 and S4 (Supplementary material), all ICCs have wide overlapping confidence intervals. The confidence interval width can only be decreased by inclusion of many more $(>100)$ subjects. Only then significance of the apparent differences between ICC values may be deduced. Now, only the parameters with ICC values above 0.9 can be considered reproducible at an acceptable level. During spontaneous breathing, this only holds for CBFV in the morning and afternoon (0.93), $\mathrm{BP}$ in the morning (0.91) and RAP (0.91) in the afternoon. In paced breathing, this only holds for CBFV (0.94) and RAP (0.92) in the afternoon. None of the autoregulation parameters shows adequate reproducibility.

\section{Discussion}

Our evaluation of four different signal-processing strategies shows that there is only limited influence on dCA parameters. Spectral smoothing [16] compared to epoch averaging [30] was suggested to improve detection of very low frequency cerebral haemodynamic oscillations. Our results only show slight improvement of coherence using spectral smoothing without an effect on gain and phase. Also the smoothness priors detrending method [27] results in higher coherence in the very low frequency range with hardly any effect on gain and phase. It seems therefore that the gain and phase parameters in the frequency range from 0.04 to $0.16 \mathrm{~Hz}$ are very robust for the different signal processing methods applied. 
Impaired early morning (6-8 a.m.) cerebral autoregulation was shown compared to evening (6-8 pm) measurements in 20 healthy subjects [3]. To assess ARI, the investigators used the thigh cuff inflation-deflation method. We investigated our 19 subjects in the morning (10 a.m.) and afternoon (2 p.m.) and could not demonstrate significant differences in dCA parameters using TFA. The ARI values extracted from the transfer function neither showed morning versus afternoon differences. Based on our results, no preference can be made for morning or afternoon dCA measurements. However, we cannot rule out the possible influence on $\mathrm{dCA}$ due to circadian rhythms. Neither can we counter the morning-evening differences shown by Ainslie et al., since our morning and afternoon measurements were at different times (10 a.m. and 2 p.m.).

From the analysis of transfer function dCA parameters, it becomes clear that ICC values are very low, meaning that dCA reproducibility is poor. From the dCA parameters, ARI has the lowest ICC. Because of the poor ICCs and the low number of subjects in this study, 95\% confidence intervals for ICC are very wide and no significant differences can be found between different analysis methods, time-of-day, or breathing strategies. Earlier, Reinhard et al. compared spontaneous versus paced breathing and could not demonstrate significant differences between both methods [24]. We do find significant differences for phase and ARI parameter values comparing both breathing methods. ARI is significantly higher in paced compared to spontaneous breathing in all but method $\mathrm{I}$ in the afternoon.

In order to detect impaired cerebral autoregulation during spontaneous breathing, dCA phase should, based on our data, be less than 0.4 rad (criterion: mean $-2 \mathrm{SD}$ ). For MMPF, the criterion for dCA impairment is a phase value less than $0.2 \mathrm{rad}$ for component 3 and 0.3 for component 4 . Due to the large inter- and intrasubject variability, the application of the different methods for the evaluation of $\mathrm{dCA}$, although in good agreement, has limited value for the individual healthy subject. Whether this is also true for diseased populations remains to be investigated.

The ICCs we found for the MMPF method for phase are comparable with the reported values from $\mathrm{Hu}$ et al. [10]. Only for paced breathing, MMPF has a higher, although not significant, ICC compared to conventional TFA. In contrast with $\mathrm{Hu}$ et al., we found better ICC for ARI, whereas significance in this case cannot be determined. Their low ICC value maybe explained by the fact that $\mathrm{Hu}$ et al. calculate ARI by applying the Tiecks model only on short data segments, whereas we based the ARI on the estimated transfer function from the recording period of $15 \mathrm{~min}$. We also report here ICC values for MMPF gain and the suggested MTFA. For gain, MMPF seems to do

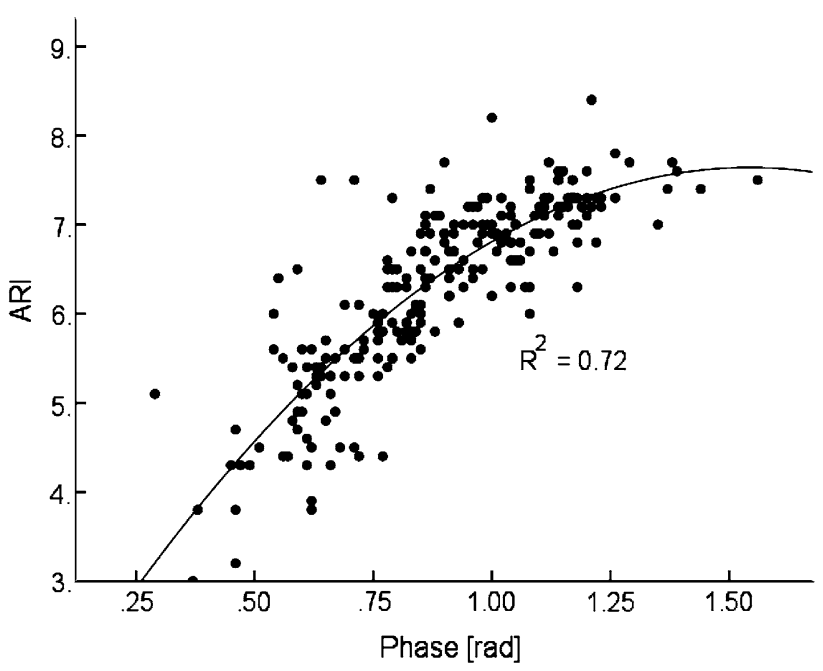

Fig. 5 Relationship between index of autoregulation (ARI) and phase during spontaneous breathing for method II

better than the TFA, but the MTFA analysis does not seem to be an improvement.

Recently, Czosnyka et al. showed good correlation $(R=0.62)$ between ARI and the time correlation based cerebral autoregulation method Mx [8]. We calculated ARI from the step response of our transfer function. Figure 5 shows the correlation between ARI and phase that was not shown before, which is even better $\left(R^{2}=0.72\right)$. However, these correlations do not imply that $\mathrm{dCA}$ parameters reproduce well. Our low ICC values for ARI are comparable to the findings of Brodie et al., while our ICCs for RAP show much higher values (ranging 0.86-0.92 vs. 0.70-0.75) [5]. The reason for this difference is not clear. Brodie estimates RAP from the first harmonic of BP and CBFV [2], whereas we performed linear regression between BP and CBFV waves [19]. The possible influence of the analysis method with regard to this needs to be elaborated further. The high ICC values for reproducibility of RAP are promising and could open directions to a more robust parameter to estimate dCA. Although RAP in itself is not a measure of $\mathrm{dCA}$, the relation of variations in $\mathrm{RAP}$ in relation with $\mathrm{ABP}$ changes may be worthwhile to investigate.

Only in 9 of the 19 subjects end-tidal $\mathrm{pCO}_{2}$ was recorded. Therefore, no results could be given for the whole group. As was shown by Reinhard et al. [24], especially during paced breathing, where the subjects are likely to increase the tidal volumes influence of $\mathrm{pCO}_{2}$ can be seen. $\mathrm{pCO}_{2}$ drops after the start of paced breathing resulting in lower CBFV. From our results, no benefits arise in favour of paced breathing versus spontaneous breathing.

In this study, no impact of the different signal processing approaches on dCA parameters was found. It is shown 
that gain and phase are very robust under the different analysis methods evaluated, and there is no need for prescribing a specific one of these signal-processing regimes. The threshold for significant coherence is not a fixed value, but depends on the degrees of freedom of each approach. Based on reproducibility results, no preference can be made for morning versus afternoon measurements or for spontaneous versus paced breathing. Poor reproducibility of dCA parameters remains a major issue hampering the clinical applicability in individual patients. Future research should be directed towards less variable dCA parameters. RAP could be a promising parameter to further explore in developing methods for estimating dCA.

In conclusion, quantification of dCA does not favour TFA versus MMPF in terms of reproducibility, which is poor for both gain and phase. Neither does it favour as a stimulus spontaneous versus paced breathing and morning versus afternoon day-time recording.

Acknowledgments The authors thank Prof. R.B. Panerai (University of Leicester, UK) for his valuable contributions to the manuscript.

Open Access This article is distributed under the terms of the Creative Commons Attribution Noncommercial License which permits any noncommercial use, distribution, and reproduction in any medium, provided the original author(s) and source are credited.

\section{References}

1. Aaslid R, Lindegaard KF, Sorteberg W, Nornes H (1989) Cerebral autoregulation dynamics in humans. Stroke 20:45-52

2. Aaslid R, Lash SR, Bardy GH, Gild WH, Newell DW (2003) Dynamic pressure-flow velocity relationships in the human cerebral circulation. Stroke 34:1645-1649

3. Ainslie PN, Murrell C, Peebles K, Swart M, Skinner MA, Williams MJ, Taylor RD (2007) Early morning impairment in cerebral autoregulation and cerebrovascular $\mathrm{CO} 2$ reactivity in healthy humans: relation to endothelial function. Exp Physiol 92:769-777

4. Blaber AP, Bondar RL, Stein F, Dunphy PT, Moradshahi P, Kassam MS, Freeman R (1997) Transfer function analysis of cerebral autoregulation dynamics in autonomic failure patients. Stroke 28:1686-1692

5. Brodie FG, Atkins ER, Robinson TG, Panerai RB (2009) Reliability of dynamic cerebral autoregulation measurement using spontaneous fluctuations in blood pressure. Clin Sci (Lond) 116:513-520

6. Claassen JA, Levine BD, Zhang R (2009) Dynamic cerebral autoregulation during repeated squat-stand maneuvers. J Appl Physiol 106:153-160

7. Cronbach LJ, Gleser GC, Nanda H, Rjaratnam N (1972) The dependability of behavioral measurements: theory of generalizability for scores and profiles. Wiley, New York

8. Czosnyka M, Smielewski P, Lavinio A, Pickard JD, Panerai R (2008) An assessment of dynamic autoregulation from spontaneous fluctuations of cerebral blood flow velocity: a comparison of two models, index of autoregulation and mean flow index. Anesth Analg 106:234-239
9. Diehl RR, Linden D, Lucke D, Berlit P (1995) Phase relationship between cerebral blood flow velocity and blood pressure. A clinical test of autoregulation. Stroke 26:1801-1804

10. Hu K, Peng CK, Czosnyka M, Zhao P, Novak V (2008) Nonlinear assessment of cerebral autoregulation from spontaneous blood pressure and cerebral blood flow fluctuations. Cardiovasc Eng 8:60-71

11. Liau BY, Yeh SJ, Chiu CC, Tsai YC (2008) Dynamic cerebral autoregulation assessment using chaotic analysis in diabetic autonomic neuropathy. Med Biol Eng Comput 46:1-9

12. Lo MT, Hu K, Liu Y, Peng CK, Novak V (2008) Multimodal pressure flow analysis: application of Hilbert Huang transform in cerebral blood flow regulation. EURASIP J Appl Signal Process 2008:785243

13. Lo MT, Novak V, Peng CK, Liu Y, Hu K (2009) Nonlinear phase interaction between nonstationary signals: a comparison study of methods based on Hilbert-Huang and Fourier transforms. Phys Rev E 79:061924

14. Mahony PJ, Panerai RB, Deverson ST, Hayes PD, Evans DH (2000) Assessment of the thigh cuff technique for measurement of dynamic cerebral autoregulation. Stroke 31:476-480

15. Mitsis GD, Poulin MJ, Robbins PA, Marmarelis VZ (2004) Nonlinear modeling of the dynamic effects of arterial pressure and $\mathrm{CO} 2$ variations on cerebral blood flow in healthy humans. IEEE Trans Biomed Eng 51:1932-1943

16. Muller T, Reinhard M, Oehm E, Hetzel A, Timmer J (2003) Detection of very low-frequency oscillations of cerebral haemodynamics is influenced by data detrending. Med Biol Eng Comput 41:69-74

17. Newell DW, Aaslid R, Lam A, Mayberg TS, Winn HR (1994) Comparison of flow and velocity during dynamic autoregulation testing in humans. Stroke 25:793-797

18. Novak V, Yang AC, Lepicovsky L, Goldberger AL, Lipsitz LA, Peng CK (2004) Multimodal pressure-flow method to assess dynamics of cerebral autoregulation in stroke and hypertension. Biomed Eng Online 3:39

19. Panerai RB (2003) The critical closing pressure of the cerebral circulation. Med Eng Phys 25:621-632

20. Panerai RB, Dawson SL, Potter JF (1999) Linear and nonlinear analysis of human dynamic cerebral autoregulation. Am J Physiol 277:H1089-H1099

21. Panerai RB, Eames PJ, Potter JF (2003) Variability of timedomain indices of dynamic cerebral autoregulation. Physiol Meas 24:367-381

22. Paulson OB, Strandgaard S, Edvinsson L (1990) Cerebral autoregulation. Cerebrovasc Brain Metab Rev 2:161-192

23. Portney LG, Watkins MP (1999) Foundations of clinical research: applications to practice. Prentice Hall, Norwalk, CT

24. Reinhard M, Muller T, Guschlbauer B, Timmer J, Hetzel A (2003) Transfer function analysis for clinical evaluation of dynamic cerebral autoregulation-a comparison between spontaneous and respiratory-induced oscillations. Physiol Meas 24:27-43

25. Simpson DM, Panerai RB, Ramos EG, Lopes JM, Marinatto MN, Nadal J, Evans DH (2004) Assessing blood flow control through a bootstrap method. IEEE Trans Biomed Eng 51: 1284-1286

26. Streiner DL, Norman GR (1995) Health measurement scales: a practical guide to their development and use, 2nd edn. Oxford University Press, Oxford

27. Tarvainen MP, Ranta-Aho PO, Karjalainen PA (2002) An advanced detrending method with application to HRV analysis. IEEE Trans Biomed Eng 49:172-175

28. Tiecks FP, Lam AM, Aaslid R, Newell DW (1995) Comparison of static and dynamic cerebral autoregulation measurements. Stroke 26:1014-1019 
29. Timmer J, Lauk M, Deuschl G (1996) Quantitative analysis of tremor time series. Electroencephalogr Clin Neurophysiol 101: 461-468

30. Welch PD (1967) The use of fast Fourier transform for the estimation of power spectra: a method based on time averaging over short, modified periodograms. IEEE Trans Audio Electroacoust 15:70-73

31. Zhang R, Zuckerman JH, Giller CA, Levine BD (1998) Transfer function analysis of dynamic cerebral autoregulation in humans. Am J Physiol 274:H233-H241 